\title{
Cone Beam Computed Tomography - Applications in Orthodontics
}

\author{
Dr Sweta Singh,' Dr Shikha Singh,,2 Dr Gautam Singh,3 Dr Amit Prakash \\ 'Senior Lecturer, ${ }^{2}$ Reader, Dept of Orthodontics, ${ }^{2}$ Senior Lecturer, Dept of Conservative Dentistry, \\ People's College of Dental Sciences, Bhopal, India \\ ${ }^{3}$ Senior Lecturer, Dept of Orthodontics, Sri Aurbindo College of Dental Sciences, Indore, India \\ Correspondence: Dr Amit Prakash; Email: amitprakash3o@gmail.com
}

\section{INTRODUCTION}

The introduction of Cone Beam Computed Tomography (CBCT) in dentistry has made it probable to visualize the dentition, the maxillofacial skeleton, and the relationship of anatomic structures in various dimensions.

As the beam covers the entire region of interest, $x$ ray source makes one pass or less around the patient's head, when acquiring images. The beam is captured on a two dimensional planar detector. The beam diameter ranges from $4 \mathrm{~cm}$ to 30 $\mathrm{cm}$. The sensor captures from 159 to 600 basis images. These images then compute a spherical or cylindrical volume of the area. In this volume, the densities at all locations (voxels) are calculated from the basis images. Voxels are cuboids and can generate images in the axial, saggital and coronal planes. The processed volumetric data are exported from the CBCT manufacturer's software as a Digital Imaging and Communications in Medicine (DICOM) files. From this data set operator can extract thick or thin, planar or curved reconstructions in any orientation.

Field of View of $\mathrm{CBCT}$ is preferred over large volume CBCT for the following reasons:

1. Increased resolution to improve the diagnostic accuracy.

2. Highest possible resolution.

3. Decreased radiation exposure to the patient.

4. Time savings due to smaller volume to be interpreted.

5. Smaller area of responsibility.

6. Focus on anatomical area of interest.

\section{How to reduce radiation dose}

ALARA is the acronym for 'As Low As Reasonably Achievable' and is a fundamental principle for diagnostic radiology. ${ }^{2}$ Radiation exposures are converted to effective doses, measured in Sieverts. In the calculation, the radiation dose to specific tissue is measured, adjusted for amount of that tissue in the field of view, and weighted based on radiation sensitivity of the tissue. The weighted tissue is then summed up to produce the effective dose. The tissue used to calculate the effective dose is specified by the International Commission on Radiological Protection (ICRP). ${ }^{3}$

It can also be achieved by:

1. Following appropriate radiograph selection criteria after taking a history from the patient, then clinical evaluation by an appropriate health care professional.

2. The use of properly trained and credentialed personnel to make radiographic exposures upon the prescription of a licensed health care professional.

3. The use of optimal technique factors including beam projection geometry, beam energy, collimation and filtration.

4. Use of the fastest $x$ - ray detector consistent with obtaining a radiographic image of optimum diagnostic quality. ${ }^{4}$

\section{Application of CBCT in orthodontics}

It should be limited to the assessment and treatment of complex anatomic conditions such as:

1. Identification of impacted canines and other impacted teeth

2. Diagnosis of root resorption as it occurs generally on the facial and lingual side of the tooth which is difficult to quantify with two dimensional views

3. Fractured roots

4. Orthodontic temporary anchorage device placement

5. Asymmetry evaluation

6. Temporomandibular joint degenerative changes

7. Cleft lip and palate

8. Soft tissue and airway assessment

9. Virtual models ${ }^{3}$

\section{Limitations of $\mathrm{CBCT}$}

While clinical applications of CBCT have increased, it has limitations related to the cone beam projection in geometry, 
detector sensitivity and contrast resolution that produces images that lack the clarity. The clarity of $\mathrm{CBCT}$ images is affected by artifacts, noise and poor soft tissue contrast. ${ }^{5}$

\section{Artifacts associated with CBCT}

Artifact can be described as visualized structure in the data that is not present in the object under investigation. These are induced mainly due to difference between the actual physical conditions of the set up that are associated with CBCT scanner's technical composition and the position of the object under investigation and the mathematical assumptions used for 3-D reconstruction. ${ }^{6}$

Various artifacts on the basis of their cause are:

1. Xray beam artifacts arise from the inherent polychromatic nature of the projection $x$ ray beam that results in beam hardening which results in two types of artifacts distortion of metallic structures due to differential absorption known as a cupping artifact and streaks and dark bands that can appear between two dense objects.?

2. Patient related artifacts are caused by patient motion which appears as unsharpness in reconstructed images. It can be minimized by using a head restraint and a short scan time. ${ }^{8}$

3. Scanner related artifacts appear as circular or ring shaped resulting from imperfection in scanner detection or poor calibration.

4. Cone beam related artifacts

a. Partial volume averaging which is a feature of conventional fan and $\mathrm{CBCT}$ imaging. It occurs due to greater resolution of scan than the contrast resolution of the object to be imaged. It can be reduced by selection of voxel.

b. Undersampling occurs when too few basis projections are provided for the reconstruction. A reduced data sample leads to misregistration, sharp edges and noisier images. It can be reduced by maintaining the number of basis projection images. ${ }^{9}$

5. Cone beam effect seen in periapical portions of scan volume. It happens due to the divergence of the $x$ ray beam as it rotates around the patient in a horizontal plane. The total amount of information is reduced because the outer row detector pixels record less attenuation, whereas more information is recorded for objects projected on the more central detector pixels, which results in image distortion, streaking artifacts and increased peripheral noise. It can be minimized by incorporating various forms of cone beam reconstructions. Clinically it can be reduced by positioning the region of interest adjacent to the horizontal plane of the $x$ ray beam and collimation of the beam to an appropriate field of view.

6. Image noise is produced when scattered radiation is recorded by pixels on detector which does not reflect the actual attenuation of object within a specific path of the $x$ ray beam. ${ }^{10}$

7. Poor soft tissue contrast.

\section{DISCUSSION}

The long awaited incorporation of the third dimension to our radiographic records is now a reality. CBCT is considered as an important tool in the field of diagnosis and treatment planning but the operator must also take care not to overlook the radiation exposure that is caused and so usage in the patient must be justified. The knowledge of risk and benefits must be assessed prior to the use. Although considerable technical efforts are put forward to reduce the artifacts caused but operator must be aware of these before concluding the final diagnosis.

\section{REFERENCES}

1. White SC, Pharoh MJ. The evolution and application of dental maxillofacial imaging modalities. Dent Clin N Am. 2008:689-705.

2. Farman AG. ALARA still applies. Oral Surg Oral Med Oral Path Oral Radiol Endod. 2005; 100:395-7.

3. Hechler SL. Cone Beam CT: Applications in Orthodontics. Dent Clin N Am. 52 2008: 809-23.

4. Scarfe WC, Farman AG, Ludlow JB, Lester WS, See M, Bailey LJ, Hershey HG. What is cone beam CT and how does it work? Dent Clin N Am. 2008; 52:707-30.

5. Scarfe WC, Farman AG, Sukovic P. Clinical applications of cone beam computed tomography in dental practice. J Canadian Dent Assoc. 2006; 72:75-80.

6. Katsumata A, Hirukawa A, Noujeim M, Okumura S, Naitoh M, Fujishita M, Ariji E, Langlais RP. Image artifact in dental cone beam CT. Oral Surg Oral Med Oral Path Oral Radiol Endod. 2006; 101:652-7.

7. Barrett JF, Keat N. Radiographic artifacts in CT: Recognition and avoidance. Radiographics. 2004; 24:1679-91.

8. Yu L, Pan X, Peliizari CA. Image reconstruction with a shift-variant filtration in circular cone-beam CT. Int J Imaging Syst Technol. 2005; $14: 213-21$.

9. Zhang Y, Zhang L, Zhu XR, Lee AK, Chambers M, Dong L. Reducing metal artifacts in cone-beam CT images by preprocessing projection data. Int J Radiat Oncol Biol Phys. 2007; 67:924-32.

10. Kyriakou Y, Prell D, Kalender WA. Ring artifact correction for high-resolution micro CT. Phys Med Biol. 2009; 54:385-91. 